\title{
Complicações anestésicas em Cirurgia Plástica e a importância da consulta pré-anestésica como instrumento de segurança
}

\author{
Preanesthetic evaluation: instrument for safety and prevention of Plastic Surgery \\ anesthesia perioperative complications
}

\author{
Ulises Prieto y \\ SCHWARTZMAN $^{1}$ \\ Kátia Torres Batista ${ }^{2}$ \\ Leonardo Teixeira D. \\ DuARTE $^{3}$ \\ Renato Ângelo Saraiva ${ }^{4}$ \\ Maria do Carmo Barreto \\ De C. Fernandes ${ }^{1}$
}

Trabalho realizado no Hospital Sarah Brasília, Brasília, DF, Brasil.

Artigo submetido no SGP (Sistema de Gestão de Publicações) da RBCP.

Artigo recebido: $25 / 2 / 2011$

Artigo aceito: 18/6/2011

\begin{abstract}
RESUMO
Introdução: A anestesia para procedimentos de Cirurgia Plástica eletivos é geralmente muito segura, todavia o conhecimento das complicações mais frequentes e o controle efetivo são importantes para a segurança do paciente. A consulta pré-anestésica realizada por equipe formada por anestesista e enfermeiros já está estabelecida no contexto da segurança do paciente cirúrgico, pois pode reduzir as intercorrências intra e pós-operatórias e evitar desfechos desfavoráveis. Embora tenha custos efetivos e necessite mudanças de condutas, a consulta pré-anestésica já é uma recomendação do Conselho Federal de Medicina desde 2006. Os autores descrevem as complicações anestésicas em Cirurgia Plástica observadas, ao longo de um ano, no Hospital Sarah Brasília e contextualizam a importância da consulta pré-anestésica. Método: Realizou-se estudo de coorte retrospectiva e analítica de pacientes hospitalares, focado na causalidade abordando as complicações anestésicas dos procedimentos cirúrgicos realizados pela equipe da Cirurgia Plástica e outras especialidades no Hospital Sarah Brasília. Descreveu-se a consulta anestésica realizada rotineiramente no préoperatório. Resultados: Observaram-se 14 (8\%) casos, 11 homens e 3 mulheres, de 477 pacientes operados para procedimentos reconstrutivos pela equipe de Cirurgia Plástica entre 2006 e 2007. Doze dos pacientes apresentavam co-morbidades. As principais complicações foram hipertensão e hipotensão com desfecho favorável. Conclusão: No presente estudo, a incidência de complicações anestésicas foi de $8 \%$, principalmente cardiovasculares e todas apresentaram desfecho favorável.

Descritores: Anestesia. Anestesia/efeitos adversos. Cuidados Pré-Operatórios.
\end{abstract}

\begin{abstract}
Introduction: Anesthesia for elective Plastic Surgery procedures is generally very safe, however the knowledge of the most common and effective control are important for patient safety. The authors consider the importance of pre-anesthetic performed by team of anesthesiologist and nurses in the context of patient safety in plastic surgery because it can reduce intra and postoperative outcomes and reduce complications. The anesthesia is a new trend in improving the quality of surgical patient safety. Although cost effective and require behavioral changes is already a recommendation of the Federal Council of Medicine since 2006. The authors describe the anesthetic complications observed in Plastic Surgery over a year at Sarah Hospital Brasilia and describe the pre-anesthetic evaluation importance of the relationship between pre-anesthetic and anesthetic complications. Methods: This is part of a retrospective cohort study of hospital patients and analytical, focused on causality anesthetic complications of surgical procedures performed in the Sarah Hospital in Brasilia. We describe the anesthetic consultation routinely performed preoperatively. Results: There were 14 (8\%) cases, 11 men and 3 women of 477 patients who performed reconstructive procedures between 2006 and 2007. Twelve patients have comorbidities. Major complications were hypotension and hypertension with favorable outcome. Conclusion: This study was low incidence of anesthetic complications and all showed favorable outcome.
\end{abstract}

Keywords: Anesthesia. Anesthesa/adverse effects. Preoperative Care.

\footnotetext{
1. Mestre em Ciências da Reabilitação, Enfermeiro do Hospital Sarah Brasília, Brasília, DF, Brasil.

2. Membro titular da Sociedade Brasileira de Cirurgia Plástica; Médica do Hospital Sarah Brasília, Brasília, DF, Brasil.

3. Mestre em Ciências da Reabilitação; Médico Anestesiologista da São Paulo Serviços Médicos de Anestesia do Hospital Sírio Libanês; Título superior em Anestesiologia, São Paulo, SP, Brasil.

4. Doutor em Anestesiologia; Médico anestesiologista do Hospital Sarah Brasília, Brasília, DF, Brasil.
} 


\section{INTRODUÇÃO}

Existe uma tendência mundial para maximizar os resultados cirúrgicos, a satisfação do paciente e reduzir os riscos nos procedimentos anestésicos e cirúrgicos. A anestesia geral e o bloqueio regional são ainda a principal opção para a maioria dos procedimentos cirurgicos, mas têm riscos conhecidos.

Embora seja geralmente muito segura, a cirurgia plástica eletiva apresenta taxa de mortalidade de cerca de $1 \mathrm{em}$ 50.000 , com $2 / 3$ destas mortes relacionadas a complicações respiratórias decorrentes de trombose venosa e embolia pulmonar, havendo necessidade do uso de anticoagulação em pacientes classificados como de risco e, consequentemente, aumentando o risco de complicações hemorrágicas ${ }^{1}$.

É senso comum que o paciente necessita da avaliação pré-operatória com anamnese, exame físico e complementares, quando pertinentes, antes de ser submetido à anestesia. Estudos recentes demonstram os benefícios da avaliação anestésica realizada antes da cirurgia em comparação àquelas realizadas no pré-operatório imediato ${ }^{1-4}$. $\mathrm{Na}$ consulta préanestésica, ao se avaliar o paciente que será submetido à anestesia, aumenta-se a chance de resultado favorável e reduz-se o risco de complicações.

No Brasil, os consultórios para avaliação pré-anestésica e clínicas com esta finalidade têm aumentado e já existe a recomendação do Conselho Federal de Medicina (CFM) por meio da Resolução $\mathrm{n}^{\mathrm{o}} 1.802 / 06^{5}$. Porém, ainda há carência deste tipo de atendimento e cuidado em muitos serviços de saúde ${ }^{1,4}$. Ademais, há estudos que procuram esclarecer quais as complicações podem ser evitadas ou previstas, a fim aumentar a segurança do procedimento anestésico e reduzir a morbidade, o tempo de internação e os gastos ${ }^{6}$.

Neste artigo, descreveram-se as complicações anestésicas em Cirurgia Plástica no Hospital Sarah Brasília e contextualizou-se a importância da consulta pré-anestésica na relação entre a avaliação pré-anestésica e complicações anestésicas.

\section{MÉTODO}

Trata-se de parte de um estudo de coorte retrospectivo e analítico de pacientes hospitalares, focado na causalidade, abordando as complicações anestésicas dos procedimentos cirúrgicos realizados no Hospital Sarah Brasília.

No pré-operatório, realizou-se rotineiramente a consulta pré-anestésica com a avaliação clínica, exames pré-operatórios, consulta com outros especialistas, sempre que necessário, definição do plano anestésico e instrução quanto ao ato anestésico, à medicação pré-anestésica, à analgesia pós-operatória e eventual internação em unidade de terapia intensiva após a cirurgia.

Todas as informações colhidas durante a consulta préanestésica (Quadro 1) e a concordância do paciente foram
Quadro 1 - Principais informações obtidas na consulta pré-anestésica no Hospital Sarah.

\begin{tabular}{l}
\hline Identificação \\
\hline Dados antropométricos \\
\hline Cirurgia proposta \\
\hline $\begin{array}{l}\text { Antecedentes pessoais: doenças pré-existentes, infecções, uso de } \\
\text { medicamentos e drogas }\end{array}$ \\
\hline Antecedentes familiares \\
\hline Cirurgias e anestesias prévias; alergias \\
\hline Sinais vitais; inspeção da pele \\
\hline Classificação ASA (American Society of Anesthesiologists)* \\
\hline Avaliação das vias aéreas utilizando-se a classificação de \\
Mallampati** \\
\hline Exames complementares \\
\hline Recomendação de jejum pré-operatório*** \\
\hline
\end{tabular}

registradas em prontuário. $\mathrm{O}$ consentimento informado por escrito preciso, atualizado e confidencial foi assinado pelo paciente e anexado ao prontuário. Os Quadros 2 e 3 apresentam, respectivamente, a Classificação do estado físico segundo a American Society of Anesthesiologists (ASA) e a Classificação de Mallampati para avaliação das vias aéreas. $A$ recomendação do tempo mínimo de jejum segundo a ASA é apresentada no Quadro 4.

Quadro 2 - Classificação do estado físico segundo a ASA.

\begin{tabular}{l|l}
\hline ASA 1 & Paciente saudável \\
\hline ASA 2 & Paciente com doença sistêmica leve \\
\hline ASA 3 & Paciente com doença sistêmica grave \\
\hline ASA 4 & $\begin{array}{l}\text { Paciente com doença sistêmica grave que é uma } \\
\text { ameaça constante à vida }\end{array}$ \\
\hline ASA 5 & $\begin{array}{l}\text { Paciente moribundo que não se espera que sobreviva } \\
\text { sem a cirurgia }\end{array}$ \\
\hline ASA 6 & $\begin{array}{l}\text { Paciente com morte cerebral cujos órgãos serão } \\
\text { removidos para fins de doação }\end{array}$ \\
\hline
\end{tabular}

Fonte: American Society of Anesthesiologists, 2010.

\begin{tabular}{l|l}
\hline \multicolumn{2}{c}{ Quadro 3 - Classificação de Mallampati. } \\
\hline Mallampati classe 1 & $\begin{array}{l}\text { Palato mole, fauces, úvula e pilares } \\
\text { visíveis }\end{array}$ \\
\hline Mallampati classe 2 & Palato mole, fauces e úvula visíveis \\
\hline Mallampati classe 3 & Palato mole e base da úvula visível \\
\hline Mallampati classe 4 & Apenas palato duro \\
\hline Fonte: Sociedade Brasileira de Anestesiologia, 2006.
\end{tabular}

Fonte: Sociedade Brasileira de Anestesiologia, 2006. 


\begin{tabular}{l|c}
\hline \multicolumn{2}{c}{ Quadro 4 - Recomendação de jejum segundo a ASA. } \\
\hline Tipo de alimento & Tempo mínimo de jejum \\
\hline Liquido sem resíduo & 2 horas \\
\hline Leite materno & 4 horas \\
\hline Fórmula infantil & 6 horas \\
\hline Leite não materno & 6 horas \\
\hline Dieta leve & 6 horas \\
\hline Carnes e frituras & 8 horas \\
\hline \multicolumn{2}{l}{ Fonte: Serviço de Anestesiologia do HC-USP-Ribeirão. }
\end{tabular}

Foram incluídos no estudo os pacientes que apresentaram algum tipo de complicação relacionada à anestesia para procedimento cirúrgico, entre abril de 2006 e dezembro de 2007, independentemente de fatores como classificação do estado físico, idade, sexo, tipo de cirurgia, tempo de anestesia, tipo de anestesia, doenças pré-existentes, entre outros.

Foi realizada, inicialmente, análise de regressão logística condicional univariada ${ }^{7}$; aquelas que apresentaram $p$-valor $<$ 0,257 foram selecionadas para serem incluídas como covariáveis na análise multivariada. Os modelos foram construídos pela exclusão consecutiva de cada variável oriunda do modelo completo composto pelas variáveis selecionadas da análise univariada, usando o teste da razão de verossimilhança ${ }^{7}$.

Para cada caso que complicou, foram selecionados três controles que não complicaram na mesma especialidade, conforme as variáveis para análise, e correlação com as complicações anestésicas: sexo e idade do paciente; estado físico (ASA) no pré-anestésico; especialidade médica; tipo de anestesia (geral-venosa, inalatória, regional - bloqueios de ramos ou plexos, espinhal - ou combinada - associação de mais de uma técnica); classificação da complicação por gravidade (leve, moderada e grave); comorbidades e hábitos de vida apresentados pelo paciente; data da realização da consulta pré-anestésica e data da cirurgia.

A análise dos dados coletados foi armazenada e analisada através dos programas Excel e programa estatístico SPSS 13.0.

\section{RESULTADOS}

Entre abril de 2006 e dezembro de 2007, foram realizados 6365 procedimentos anestesicos no Hospital Sarah Brasília, destes, 175 apresentaram algum tipo de complicação relacionada à anestesia, com taxa de $2,74 \%$ (Tabela 1 ). A complicação leve esteve presente em $61 \%$ dos casos.

Para comparação, foi selecionada amostra estratificada aleatória proporcional, pareada para cada caso, 3 a 4 controles segundo especialidade cirúrgica, sexo e estado físico, totalizando 700 pacientes estudados. A distribuição dos pacientes por especialidade médica foi dividida em 5 clínicas cirúrgicas

\begin{tabular}{c|c|c|c}
\hline \multicolumn{3}{c}{$\begin{array}{r}\text { Tabela } 1 \text { - Distribuição dos pacientes casos e } \\
\text { controles por especialidade médica. }\end{array}$} \\
\cline { 1 - 3 } Especialidade & \multicolumn{2}{c}{ Complicação } & $\begin{array}{c}\text { Total } \\
\text { n (\%) }\end{array}$ \\
\cline { 2 - 4 } & $\begin{array}{c}\text { Não } \\
\text { n (\%) }\end{array}$ & $\begin{array}{c}\text { Sim } \\
\text { n (\%) }\end{array}$ & \\
\hline $\begin{array}{c}\text { Cirurgia } \\
\text { Plástica }\end{array}$ & $38(7,2 \%)$ & $14(8,0 \%)$ & $52(7,5 \%)$ \\
\hline Neurocirurgia & $63(12,0 \%)$ & $43(24,6 \%)$ & $106(15,1 \%)$ \\
\hline Ortopedia & $298(56,8 \%)$ & $81(46,2 \%)$ & $379(54,2 \%)$ \\
\hline $\begin{array}{c}\text { Cirurgia } \\
\text { Torácica }\end{array}$ & $73(13,9 \%)$ & $19(10,9 \%)$ & $92(13,1 \%)$ \\
\hline Urologia & $53(10,1 \%)$ & $18(10,3 \%)$ & $71(10,1 \%)$ \\
\hline Total & $525(100,0 \%)$ & $175(100,0 \%)$ & $700(100,0 \%)$ \\
\hline
\end{tabular}

Fonte: CNCQ Sarah- Brasília ${ }^{8}$.

que realizaram o ato operatório: Cirurgia Plástica, Neurocirurgia, Ortopedia, Cirurgia Torácica e Urologia (Tabela 2).

A equipe da Cirurgia Plástica foi representada com o menor número de pacientes operados (477 casos; 7,5\%) e menor número de complicações decorrentes da anestesia (14 casos; 8\%) quando comparada às diversas especialidades, ao longo de um ano. Os procedimentos realizados pela Cirurgia Plástica no Hospital Sarah incluíram procedimentos complexos para reconstrução de membro superior (lesões neurotendíneas, tumores), membro inferior, lesões no tronco (úlceras por pressão, mielomeningocele, ostemilielite) (Figuras 1 a 3).

Os pacientes operados pela Cirurgia Plástica que apresentaram complicações anestésicas estão representados na Tabela 3. As principais complicações foram hipertensão e hipotensão, encontradas em oito pacientes com idade entre 45-65 anos, a média de duração dos procedimentos foi de 1 hora e 30 minutos, o procedimento anestésico foi a anestesia geral e combinada, sete destes apresentavam hipertensão arterial e o diabetes mellitus como co-morbidades. Todos os casos de complicações foram tratados durante o ato anestésico e evoluíram favoravelmente.

Nos resultados gerais do estudo, encontraram-se como fatores de risco para complicações anestésicas o estado fisico ASA, as comorbidades doenças da tiroide, respiratórias, tumores ou doença pulmonar, ex-fumantes e duração da anestesia.

\section{DISCUSSÃO}

Os procedimentos de Cirurgia Plástica realizados no Hospital Sarah podem ser de caráter eletivo ou de urgência, e principalmente procedimentos de reconstrução. O tempo de duração dos procedimentos variou de uma a cinco horas, em 
Tabela 2 - Relação de pacientes submetidos a procedimentos pela equipe de Cirurgia Plástica que apresentaram complicações anestésicas.

\begin{tabular}{|c|c|c|c|c|c|}
\hline Idade & Comorbidade & Procedimento & Anestesia & $\begin{array}{l}\text { Duração da } \\
\text { cirurgia }\end{array}$ & $\begin{array}{c}\text { Complicação } \\
\text { Anestésica }\end{array}$ \\
\hline $40 / F$ & $\mathrm{HA} / \mathrm{DM}$ & $\begin{array}{l}\text { Abertura polia A1 do } \\
\text { polegar }\end{array}$ & Combinada & $1 \mathrm{~h} 25^{\prime}$ & Hipertensão \\
\hline $45 / \mathrm{M}$ & $\begin{array}{l}\text { DN/ETIL/TABAG/ } \\
\text { hemotransfusão }\end{array}$ & $\begin{array}{l}\text { Fechamento de escara } \\
\text { com retalho glúteo }\end{array}$ & Geral & $5 \mathrm{~h}$ & Hipertensão \\
\hline $54 / \mathrm{M}$ & $\begin{array}{l}\text { Hipotiroidismo/ } \\
\text { meningioma/HA }\end{array}$ & $\begin{array}{l}\text { Exérese de lipoma no } \\
\text { braço }\end{array}$ & Combinada & $1 \mathrm{~h} 42^{\prime}$ & Hipertensão \\
\hline $63 / \mathrm{F}$ & $\mathrm{DM}$ & $\begin{array}{c}\text { Abertura do I } \\
\text { compartimento dorsal do } \\
\text { punho }\end{array}$ & Combinada & $1 \mathrm{~h} 67^{\prime}$ & Vômito \\
\hline $45 / \mathrm{M}$ & Sepse & $\begin{array}{l}\text { Desbridamento de ferida } \\
\text { em coxa }\end{array}$ & Geral & $1 \mathrm{~h} 75^{\prime}$ & Hipotensão \\
\hline $53 / \mathrm{M}$ & $\begin{array}{l}\text { Displasia fibrosa } \\
\text { hipertostótica }\end{array}$ & $\begin{array}{l}\text { Desarticulação no joelho } \\
\text { para tratamento de úlcera } \\
\text { na perna }\end{array}$ & Combinada & $3 \mathrm{~h} 59^{\prime}$ & Hipotensão \\
\hline $45 / \mathrm{M}$ & $\begin{array}{l}\text { Tabagismo/ etilismo/ } \\
\text { hemo-transfusão }\end{array}$ & $\begin{array}{l}\text { Exérese de lipoma na } \\
\text { região posterior da coxa }\end{array}$ & Combinada & $1 \mathrm{~h} 75^{\prime}$ & Intubação difícil \\
\hline $40 / \mathrm{M}$ & $\begin{array}{l}\text { Paraplegia/hemo- } \\
\text { transfusão }\end{array}$ & $\begin{array}{l}\text { Fechamento de escara } \\
\text { isquiática com retalho } \\
\text { glúteo }\end{array}$ & Geral & $4 \mathrm{~h} 16^{\prime}$ & $\begin{array}{l}\text { Hiperemia fixa na } \\
\text { região zigomática } \\
\text { esquerda* }\end{array}$ \\
\hline $40 / \mathrm{M}$ & $\mathrm{DM} / \mathrm{HA}$ & $\begin{array}{c}\text { Retalho sural reverso para } \\
\text { cobertura de tendão de } \\
\text { Aquiles } \\
\end{array}$ & Combinada & $3 \mathrm{~h} 67^{\prime}$ & Hipotensão \\
\hline $40 / \mathrm{M}$ & Sequela de queimadura & $\begin{array}{l}\text { Curativo cirúrgico de } \\
\text { retalho inguinal para } \\
\text { dorso da mão }\end{array}$ & Geral & $59^{\prime}$ & Vômito \\
\hline
\end{tabular}

Apoio da face na rodilha, regrediu em 24 horas; DM=Diabetes Mellitus; HA = Hipertensão arterial.

média. Foram encontrados 14 casos de complicações anestésicas para procedimentos de cirurgia plástica, na faixa etária entre 40-60 anos, analisados entre 38 controles, ou seja, $8 \%$ das complicações apresentadas por todas as especialidades cirúrgicas do Hospital Sarah. Todas as complicações apresentadas no transoperatório evoluíram com desfecho favorável.
As comorbidades, principalmente hipertensão e diabetes, foram observadas em onze pacientes. As principais complicações foram a hipertensão e a hipotensão observadas em oito pacientes. A hipotensão e hipertensão são as complicações cardiovasculares mais frequentes no período perioperatório, como podemos observar na tabela com a relação de todas as 


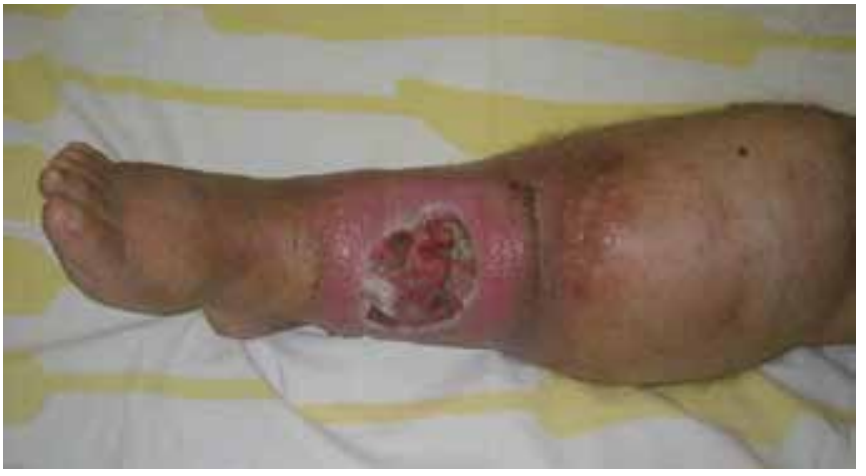

Figura 1 - Paciente do sexo masculino, 53 anos, portador de úlcera na perna por displasia fibrosa hiperostótica submeteuse a desarticulação no joelho, sob anestesia geral e peridural. Apresentou, como complicação transoperatória, hipotensão, revertida satisfatoriamente com o tratamento especifico.

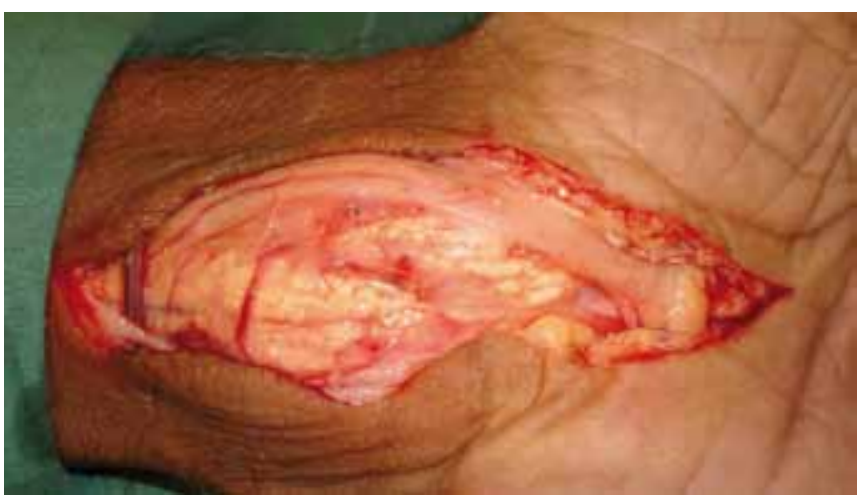

Figura 2 - Paciente do sexo masculino, 66 anos, hipertenso, submeteu-se a remoção de tofo no III QRD e cotovelo, descompressão e anteriorização do nervo ulnar, retinaculotomia dos flexores e abertura do canal de Guyon, sob anestesia regional. Apresentou hipertensão no transoperatório, revertida satisfatoriamente com o tratamento especifico.

complicações anestésicas entre as especialidades. A hipotensão pode ser decorrente de drogas anestésicas, sangramento transoperatório ou estímulo vagal. A hipertensão pode ser devida a controle insuficiente da hipertensão arterial no pré-operatório, dor ou distensão vesical ${ }^{9}$.

Nos resultados gerais do estudo, encontraram-se como fatores de risco para complicações anestésicas entre todas as especialidades cirúrgicas, o estado físico ASA, as comorbidades: doenças da tiroide, respiratórias, tumores ou doença pulmonar, ex-fumantes e duração da anestesia, conforme descrito em dissertação de mestrado ${ }^{8}$. A comparação dos resultados obtidos em outros serviços pode ser diferente desta aqui apresentada, haja vista as características dos procedimentos realizados e das doenças atendidas, que não são comuns na população geral, por se tratar de um hospital de reabilitação e de atendimento terciário ${ }^{1,10}$.

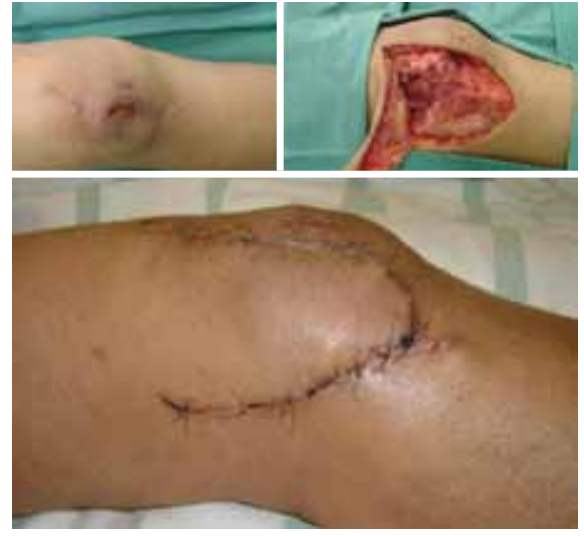

Figura 3 - Paciente do sexo feminino, 58 anos, portadora de fratura de rótula com exposição óssea, submeteu-se a retalho fasciocutâneo para o joelho direito, sob anestesia regional. Apresentou, como complicação transoperatória, a hipotensão arterial, revertida satisfatoriamente com o tratamento especifico.

\begin{tabular}{c|c|c}
\hline \multicolumn{3}{c}{ Tabela 3 - Distribuição das complicações relacionadas à } \\
anestesia em todas as especialidades cirúrgicas.
\end{tabular}

Fonte: CNCQ Sarah- Brasília ${ }^{8}$. 
Existe uma tendência mundial entre as sociedades médicas para redução de riscos e erros em cirurgias. Em 2003, a Sociedade Americana de Cirurgiões Plásticos se juntou a outras 50 grandes associações médicas e endossaram o Joint Commission Universal Protocol ${ }^{11}$, para evitar erros no procedimento e no sítio cirúrgico. A partir de 1 de julho de 2004, em todos os hospitais credenciados, atendimentos ambulatoriais e instalações cirúrgicas seriam necessários integrar diversos elementos, incluindo a identificação do paciente no pré-operatório, marcação do sítio operatório no pré-operatório e o "check-list" antes da primeira incisão. Para a Cirurgia Plástica, isso muitas vezes inclui história e exame físico precisos e atualizados, o consentimento informado e os documentos para liberação por profissionais médicos de outras especialidades, se necessário. O protocolo prevê, também, a importância de assegurar a presença de todos os materiais implantáveis necessários para o procedimento.

Ademais, outro aspecto a ser contemplado deve ser a consulta pré-anestésica. Quando analisamos os resultados obtidos neste estudo, encontramos o baixo índice de complicações relacionadas à anestesia em todos os procedimentos cirúrgicos realizados no Hospital Sarah (2,74\%), comparados aos estudos que demonstram taxas de até $18 \%{ }^{12}$. Outro dado importante se refere à não ocorrência de óbito ou sequela grave decorrentes dessas complicações, quando algumas pesquisas apontam taxas de óbito entre $0,13 \%$ e 1,5\%, considerando-se que a probabilidade atual de morte durante a anestesia, e em decorrência dela, está estimada entre 1 para 250.000 e 1 para $300.000^{12,13}$.

Outros estudos também apontam para a importância da consulta pré-anestésica, por exemplo, na Faculdade de Medicina da Universidade de São Paulo-USP, a avaliação préanestésica permitiu diminuir o tempo para avaliação clínica, reduzir as taxas de suspensão de cirurgias de 3,3\% para 1,2\% e otimizar a realização de exames de laboratório. As complicações cardiológicas durante a cirurgia foram mais frequentes quando o cirurgião realizou o preparo do do paciente ${ }^{14}$. No estudo de Schiff et al. ${ }^{10}$, a consulta pré-anestésica reduziu o tempo para consulta e os custos e teve impacto positivo na educação do paciente, embora a satisfação global do paciente com o procedimento tenha se mantida inalterada. Fica para nós clara a importância do anestesiologista não só durante $\mathrm{o}$ ato anestésico, mas como membro atuante na avaliação e na preparação do paciente para o procedimento. Ademais, o conhecimento prévio das condições clínicas e comorbidades favorecem a abordagem transoperatória, inerentes a qualquer procedimento cirúrgico e a possibilidade de intervenção e desfecho favoráveis. O principal objetivo da avaliação pré-operatória é a redução da morbimortalidade no paciente cirúrgico; sua realização, de forma completa, faz parte das estratégias para detecção de fatores de risco e de prevenção de incidentes ${ }^{15}$.
Sem dúvida, a consulta pré-anestésica ambulatorial, dias antes da cirurgia, pode ser efetiva na prevenção de complicações anestésicas, na qualidade do cuidado anestésico, no tratamento das complicações no transoperatório e, principalmente, na relação médico-paciente. Infelizmente, porém, esta é uma prática ainda recente e pouco comum em muitos serviços no Brasil. Na Rede Sarah, esta medida já vem sendo adotada desde os anos 80, o que permite à equipe anestésica participar do processo decisório na realização de procedimentos cirúrgicos.

Outro aspecto que consideramos relevante é o aspecto judicial, nos Estados Unidos, uma das três principais causas de processos judiciais é a avaliação pré-anestésica negligenciada ou não realizada ${ }^{11}$. Por outro lado, há ainda dificuldades na implantação universal da avaliação pré-anestésica. Alguns desses fatores incluem a necessidade de espaço para a realização da consulta ambulatorial, a disponibilidade, capacitação, treinamento e atualização de outros profissionais da equipe assistencial, como enfermeiros, e o deslocamento de anestesiologistas do centro cirúrgico para o ambulatório, o que exigirá mudança de pensamento de todos os membros da equipe médica hospitalar.

\section{CONCLUSÃO}

No presente estudo, foi baixa a incidência de complicações anestésicas e todas apresentaram desfecho favorável. Os autores consideram a importância da consulta pré-anestésica realizada por equipe formada por anestesista e enfermeiros no contexto da segurança do paciente em Cirurgia Plástica, pois pode reduzir as intercorrâncias intra e pós-operatórias e evitar desfechos desfavoráveis.

\section{REFERÊNCIAS}

1. Mustoe TA, Buck DW $2^{\text {nd }}$, Lalonde DH. The safe management of anesthesia, sedation, and pain in plastic surgery. Plast Reconstr Surg. 2010;126(4):165e-76e.

2. Macuco MV, Macuco OC, Bedin A, Turazzi J, Castro R, Basso A. Efeito de um consultório de Anestesiologia sobre as preocupações, percepções e preferências relacionadas à anestesia: comparação entre o sexo masculino e feminino. Rev Bras Anestesiol. 1999;49(3):179-89.

3. Mathias LAST, Mathias RS. Avaliação pré-operatória: um fator de qualidade. Rev Bras Anestesiol. 1997;47(4):335-49.

4. Turazzi JC, Castro RAC, Bedin A, Macuco MV. Clínica de avaliação pré-operatória. In: Cavalcanti IL, ed. Medicina perioperatória. Rio de Janeiro:Sociedade Brasileira de Anestesiologia;2005. p.49-69.

5. Resolução CFM n ${ }^{\circ}$ 1802/2006. Disponível em: http://www.sba.com.br/ defesa/180206.asp

6. Jung LA, Cé ACO. Complicações relacionadas à anestesia. Rev Bras Anestesiol. 1986;36(6):441-6.

7. Hosmer DW, Lemeshow S. Applied logistic regression. $2^{\text {nd }}$ ed. New York:John Wiley \& Sons;2000. p.223-59.

8. Schwartzman UP. Consulta pré-anestésica: instrumento de segurança em anestesia e prevenção de complicações perioperatórias [Dissertação de mestrado]. Brasília: Centro Sarah de Formação e Pesquisa. 93p.

9. White PF, Waddle JP. Anesthesia for Plastic Surgery. In: Plastic surgery. $2^{\text {nd }}$ ed. New York:Elsevier;2011. 
10. Schiff JH, Frankenhauser S, Pritsch M, Fornaschon SA, Snyder-Ramo SA, Heal C, et al. The Anesthesia Preoperative Evaluation Clinic (APEC): a prospective randomized controlled trial assessing impact on consultation time, direct costs, patient education and satisfaction with anesthesia care. Minerva Anesthesiol. 2010;76(7):491-9.

11. Joint Comission Universal Protocol. Disponível em: www.jointcommission.org

12. Tarrac SE. A description of intraoperative and postanesthesia complication rates. J Perianesth Nurs. 2006;21(2):88-96.
13. Heine MF, Lake CL. Nature and prevention of errors in anesthesiology. J Surg Oncol. 2004;88(3):143-52.

14. Borkowski RG, Parker BM, Fitzsimmons B, Maurer WG. The incidence of cardiac related intraoperative quality indicators ambulatory/same day surgery patients and inpatients after different preoperative evaluation processes. Anesthesiology. 2001;95:A32.

15. Egbert LD, Battit GE, Turndorf H, Beecher HK. The value of the preoperative visit by and anesthetist. A study of doctor-patient rapport. JAMA. 1963;185(7):553-5.
Correspondência para: Kátia Torres Batista

SMHS Qd 501, Cjto A - Brasília, DF, Brasil - CEP 70335-901

E-mail: katiatb@terra.com.br, ulises@sarah.br 